\title{
两态反应 $\mathrm{Ni}_{2}^{+}$与 $c-\mathrm{C}_{6} \mathrm{H}_{12}$ 的机理及自旋-轨道耦合研究
}

\author{
马军 ${ }^{a}$ 李榕 ${ }^{b}$ 任道玮 ${ }^{a}$ 马禧龙 ${ }^{a}$ 朱开礼 ${ }^{a}$ 耿志远*, $a$ \\ $\left({ }^{a}\right.$ 西北师范大学 化学化工学院 甘肃省高分子材料重点实验室 生态环境相关高分子材料教育部重点实验室 甘肃 \\ 兰州 730070) \\ $\left({ }^{b}\right.$ 甘肃民族师范学院 化学与生命科学系 甘肃合作 747000)
}

\begin{abstract}
摘要 在密度泛函理论的 B3LYP 方法下, 对两态反应 $\mathrm{Ni}_{2}^{+}$与环已烷体系进行了较为系统的研究. 结果表明, 反应分别 在第一个氢迁移 $\left({ }^{4} \mathrm{IM} 1 \rightarrow{ }^{4} \mathrm{TS} 1 / 2\right)$, 第三个氢迁移 $\left({ }^{4} \mathrm{TS} 15 / 16 \rightarrow{ }^{4} \mathrm{IM} 15\right)$ 以及 $\mathrm{Ni}_{2}{ }^{+}$翻转过程 $\left({ }^{4} \mathrm{IM} 5 \rightarrow{ }^{4} \mathrm{TS} 5 / 6,{ }^{2} \mathrm{TS} 11 / 12 \rightarrow{ }^{2} \mathrm{IM} 12\right)$ 发生了二、四重态势能面的交叉, 本文运用内禀反应坐标单点垂直激发计算的方法得到势能面大致的交叉点(CP), 进一 步利用 Crossing2004 程序获得精确的最低能量交叉点(MECP). 对 MECP 附近的自旋轨道耦合(SOC) 常数进行了计算. MECP1 MECP4 处的 SOC 值分别为 $318.01,396.89,268.74$ 和 $306.67 \mathrm{~cm}^{-1}$. 较大的 SOC 值说明不同势能面间发生了有 效地跃迁并使反应沿着最低反应通道进行. 对反应通道的研究发现, 反应中同面脱氢是主要反应通道. 异面脱氢由于 翻转过程中决速步骤势垒为 $33 \mathrm{kcal} / \mathrm{mol}($ 吸热 $3 \mathrm{kcal} / \mathrm{mol}$ ), 只生成少量的异面脱氢产物. 计算结果解释了实验现象.
\end{abstract}

关键词 密度泛函理论; 自旋-轨道耦合; 反应机理; 反应势能面

\section{Mechanism and Spin-orbit Coupling Study for Two State Reaction $\mathrm{Ni}_{2}^{+}$with Cyclohexane}
Ma, Jun ${ }^{a}$
$\mathrm{Li}$ Rong $^{b}$
Ren, Kuiwei ${ }^{a}$
Ma, Xilong ${ }^{a}$
Zhu, Kaili ${ }^{a}$
Geng, Zhiyuan*,a
( ${ }^{a}$ Key Laboratory of Polymer Materials of Gansu Province, Key laboratory of Eco-Environment-Related Polymer Materials of Ministry of Education, College of Chemistry and Chemical Engineering, Northwest Normal University, Lanzhou 730070, Gansu, China)
( ${ }^{b}$ Department of Chemistry and Life Science, Gansu Normal University for Nationalities, Hezuo 747000, Gansu, China)

\begin{abstract}
Two-state reaction mechanism of $\mathrm{Ni}_{2}^{+}$with $c-\mathrm{C}_{6} \mathrm{H}_{12}$ on the quartet and doublet potential energy surfaces has been investigated at the B3LYP level. As a result, the ground state for reactants is doublet state, for products is quartet state. A further study on different potential energy surfaces indicates that the title reaction contains four potential energy surfaces crossing in doublet and quartet state, which occurs during the first hydrogen migration step $\left({ }^{4} \mathrm{IM} 1 \rightarrow{ }^{4} \mathrm{TS} 1 / 2\right)$, the third hydrogen migration step $\left({ }^{4} \mathrm{TS} 15 / 16 \rightarrow{ }^{4} \mathrm{IM} 15\right)$ and $\mathrm{Ni}_{2}^{+}$flip step $\left({ }^{4} \mathrm{IM} 5 \rightarrow{ }^{4} \mathrm{TS} 5 / 6,{ }^{2} \mathrm{TS} 11 / 12 \rightarrow{ }^{2} \mathrm{IM} 12\right)$, respectively. The first, second and third potential energy surfaces crossing are the transitions of quartet to doublet state, and the last one is doublet to quartet state. Using single-point energy calculations based on IRC of the different states to locate the approximate structure of crossing points (CPs) between potential energy surfaces, and more accurate structure of CPs, minimal energy crossing points (MECPs), have been obtained by the Crossing 2004 procedure. The spin-orbit coupling (SOC) constant was calculated to discuss the possible spin inversion processes. The values of SOC constants near the MECP1 $\sim$ MECP4 were calculated to be $318.01,396.89,268.74$ and $306.67 \mathrm{~cm}^{-1}$, respectively, the large values of SOC constants indicate the transition from one potential energy surface to another proceeding smoothly and reducing the barrier of reaction in large extent. Based on the result of our calculation, the reaction contains two different channels, one face dehydrogenation and different face dehydrogenation, the first one is confirmed as the dominant channel due to the large exothermicity. Flip process, the critical factor leading to the different face dehydrogenation, has a large barrier of $33 \mathrm{kcal} / \mathrm{mol}$ in rate limiting step $\left({ }^{2} \mathrm{IM} 9 \rightarrow^{2} \mathrm{TS} 9 / 10 \rightarrow\right.$ ${ }^{2}$ IM10) leading to a few different face dehydrogenation products. Our calculation can well explain the experiment observation.

Keywords density functional theory; spin-orbit coupling; reaction mechanism; potential energy surfaces
\end{abstract}

\section{1 引言}

能源问题一直是人类社会所面临的重大问题. 由于 太阳能、氢能、风能等能源的有效利用, 这些清洁能源
引起了科学家们的关注. 环己烷 $\left(c-\mathrm{C}_{6} \mathrm{H}_{12}\right)$ 作为一种优秀 的储氢材料 ${ }^{[1]}$, 其脱氢机理一直是具有挑战性的课题. 环已烷在过渡金属催化下发生的脱氢反应可以分为两

* E-mail: zhiyuangeng@126.com

Received February 10, 2015; published March 30, 2015.

Supporting information for this article is available free of charge via the Internet at http://sioc-journal.cn.

Project supported by the Natural Science Foundation of Gansu Province (No. 10710RJZA114).

项目受甘肃省自然科学基金(No. 10710RJZA114)资助. 
大类. 一类是环己烷先吸附在过渡金属表面层, 如 $\mathrm{Ni}(111), \operatorname{Pt}(111)$ 或 $\mathrm{Pt}_{3} \mathrm{Sn} / \mathrm{Pt}(111)$ 等 $^{[2 \sim 4]}$, 然后再发生脱氢 反应. 另一类是过渡金属原子、离子或原子笶在气相条 件下与环已烷发生脱氢反应 ${ }^{[5,6]}$, 由于没有金属层, 脱氢 反应可以在任意方向和位点发生, 这类研究在探索反应 立体选择性方面具有科学意义. 但是由于过渡金属的气 相反应经常表现出其独有的两态性质, 往往比其他反应 更为复杂, 过渡金属的不同电子组态对其气相反应的反 应路径有着很大的影响, 由于在反应过程中金属的基态 会在不同多重度之间发生变化, 进而会使反应发生不同 势能面的交叉. 关于气相反应的机理研究, 国内也有报 道 ${ }^{[7]}$.

2011年, Schwarz 等 ${ }^{[8]}$ 研究了过渡金属二聚体离子 $\mathrm{Ni}_{2}^{+}$和 $c-\mathrm{C}_{6} \mathrm{H}_{12}$ 的反应. 发现该反应只有 $\mathrm{C}-\mathrm{H}$ 键活化的 脱氢产物, 没有 $\mathrm{C}-\mathrm{C}$ 键活化产物. 实验中运用同位素 标记法. 即: [cis-1,2,3,4,5,6-D6]- $\mathrm{C}_{6} \mathrm{H}_{6}$. 结果发现, 产物 可以分为大量的同面脱氢产物 $2 \mathrm{H}_{2}, 3 \mathrm{H}_{2}, 2 \mathrm{D}_{2}, 3 \mathrm{D}_{2}$ 和少量 异面脱氢产物 $\left[\mathrm{H}_{2}, \mathrm{D}_{2}\right],\left[\mathrm{H}_{4}, \mathrm{D}_{2}\right],\left[\mathrm{H}_{2}, \mathrm{D}_{4}\right] . \mathrm{Schwarz}^{[8]}$ 对异面 脱氢现象提出了一种翻转机理, 见图式 1 , 他们认为反 应在发生第一分子脱氢后, $\mathrm{Ni}_{2}^{+}$会通过活化 $\mathrm{C}-\mathrm{C} \sigma$ 键从 环己烷的反应面 1 翻转到反应面 2 , 再发生后续的脱氢反 应. 由于实验检测手段的局限性，迄今为止，关于 $\mathrm{Ni}_{2}^{+}$ 的翻转机理未见报道. 本文通过密度泛函理论(DFT)研 究了 $\mathrm{Ni}_{2}^{+}$催化 $c-\mathrm{C}_{6} \mathrm{H}_{12}$ 的脱氢反应, 旨在说明详细的反应 机理. 由于反应为典型的两态反应, 不同势能面之间存 在多个交叉点. 为了更深入理解该反应的微观机理, 对 涉及到势能面交叉的最低能量交叉点(MECP)处的自 旋一轨道耦合常数(SOC)进行了计算研究.

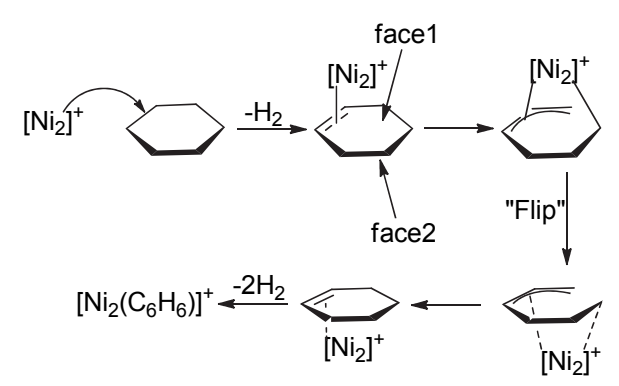

图式 1 文献 ${ }^{[8]}$ 中对异面脱氢提出的翻转过程机理图

Scheme 1 The proposed mechanism of flip process in Ref. [8]

\section{2 计算方法}

\section{1 几何构型的优化}

采用密度泛函理论中的 $\mathrm{B} 3 \mathrm{LYP}^{[9 \sim 11]}$ 方法, 对于 $\mathrm{C}$ 和 $\mathrm{H}$, 使用 6-31G**基组, 对于 $\mathrm{Ni}_{2}^{+}$, 采用考虑相对论效应 的 LANL2DZ ${ }^{[12 ~ 14]}$ 有效核势基组. 对所有反应物、产物、 中间体和过渡态的结构进行了全优化; 通过频率分析证 实了各过渡态具有唯一虚频; 用内禀反应坐标(IRC)方 法验证了反应路径. 运用了 Yoshizawa 等 ${ }^{[15]}$ 提出的内禀 坐标垂直激发单点计算的方法来确定不同自旋态下势 能面交叉点(crossing point, CP), 再通过 Harvey 等 ${ }^{[16]}$ 的 Crossing 2004 程序包, 在 CP 的基础上进一步找到对应 的 MECP. 对部分重要的中间体, 做了 NBO 自然轨道分 析. 全部计算工作运用 Gaussian $09^{[17]}$ 程序完成.

\section{2 自旋-轨道耦合常数的计算}

采用 GAMESS 程序包 ${ }^{[18]}$ 计算 MECP 附近自旋-轨 道耦合 (SOC) 常数. 基于非绝热动力学理论, 强的自旋轨道耦合作用容易使体系发生不同自旋态之间的系间 窝越. 根据单电子近似, 自旋-轨道耦合 Hamiltonian 算 符 $^{[19]}$ 表示为:

$$
\begin{aligned}
\hat{H}_{s o} & =\frac{\alpha^{2}}{2} \sum_{i} \sum_{k}\left(\frac{Z_{k}^{*}}{r_{i k}^{3}}\right)\left(\hat{S}_{i} \cdot \hat{L}_{i k}\right)=\sum_{i} h_{i}\left(Z^{*}\right) \\
\frac{\alpha^{2}}{2} & =\frac{e^{2} h}{4 \pi m_{e}^{2} c^{2}}
\end{aligned}
$$

其中, $\hat{L}_{i k}$ 是轨道角动量算符, $\hat{S}_{i}$ 是自旋轨道角动量算 符. 为了减少单电子 Hamiltonian 算符中双电子部分能 量的误差, 使用有效核电荷数 $Z_{k}^{*}$ 代替 $Z_{k}$, 来弥补相对 论效应带来的损失, $Z_{k}^{*}$ 可以从屏蔽核电荷 ${ }^{[20]}$ 中得出. 四重态和二重态旋轨耦合强度的大小取决于旋轨耦合 矩阵元, 如式(2)所示:

$$
\left\langle H_{s o}\right\rangle_{S, S^{\prime}}^{4-2}=\left\langle{ }^{4} \psi\left(M_{S}\right)\left|H_{s o}\right|^{2} \psi\left(M_{S^{\prime}}\right)\right\rangle
$$

${ }^{4} \psi$ 和 ${ }^{2} \psi$ 分别是四重态和二重态的波函数, 而 $M_{S}$ 是自 旋磁量子数. 这样四重态和二重态所产生旋轨耦合矩阵 元可通过公式(3)所给出的均方根耦合常数衡量相互作 用强度 ${ }^{[21]}$ 的大小:

$$
\begin{aligned}
& S O C^{4-2}=\left\{\left[\frac{1}{\left(2 S_{1}+1\right)\left(2 S_{2}+1\right)}\right] \times\right. \\
& \left.\sum_{S, S^{\prime}}\left[\left(\left\langle H_{s o}\right\rangle_{S, S^{\prime}}\right)_{x}^{2}+\left(\left\langle H_{S O}\right\rangle_{S, S^{\prime}}\right)_{y}^{2}+\left(\left\langle H_{s o}\right\rangle_{S, S^{\prime}}\right)_{z}^{2}\right]\right\}^{\frac{1}{2}}
\end{aligned}
$$

\section{3 结果与讨论}

为方便起见, 本文中把环已烷开始发生脱氢反应的 面定义为反应面 1 , 另一面定义为反应面 $2 . \mathrm{H}$ 原子标为 $\mathrm{H} m n$ ( $m=1 \sim 6, n=1 \sim 2), m$ 表示 $\mathrm{H}$ 所属的C原子的编号, $n=1$ 表示 $\mathrm{H}$ 位于反应面 $1, n=2$ 表示 $\mathrm{H}$ 位于反应面 2 . 对于 $\mathrm{Ni}_{2}^{+}$而言, $\mathrm{Ni} 1$ 表示开始反应时直接与 $c-\mathrm{C}_{6} \mathrm{H}_{12}$ 相连的 $\mathrm{Ni}$ 原子, $\mathrm{Ni} 2$ 则表示处于末端位置的 $\mathrm{Ni}$ 原子. 例如, H32表 示 $\mathrm{C} 3$ 上处于面 2 的 $\mathrm{H}$.

\section{1 反应物电子结构}

$\mathrm{Ni}_{2}^{+}$存在多个自旋态, 其中能量较低的自旋态为二 重态和四重态, 其电子排布分别为 $\left(\sigma_{\mathrm{g}}\right)^{2}\left(\pi_{\mathrm{u}}\right)^{4}\left(\sigma_{\mathrm{g}}\right)^{2}\left(\delta_{\mathrm{g}}\right)^{4}$ $\left(\delta_{\mathrm{u}}\right)^{4}\left(\sigma_{\mathrm{u}}\right)^{2}\left(\pi_{\mathrm{g}}\right)^{1}$ 和 $\left(\sigma_{\mathrm{g}}\right)^{2}\left(\pi_{\mathrm{u}}\right)^{4}\left(\sigma_{\mathrm{g}}\right)^{2}\left(\delta_{\mathrm{g}}\right)^{4}\left(\delta_{\mathrm{u}}\right)^{4}\left(\sigma_{\mathrm{u}}\right)^{1}\left(\pi_{\mathrm{g}}\right)^{2}$. 如图 1 所 示, $\pi_{\mathrm{g}}\left(\mathrm{d}_{ \pm 1}-\mathrm{d}_{ \pm 1}\right)$ 与 $\sigma_{\mathrm{u}}\left(\mathrm{d}_{0}-\mathrm{d}_{0}\right)$ 的能级差仅为 0.002 Hartree. 这 
三个轨道可以近似为能量简并轨道, 根据洪特规则, 电 子更倾向于自旋平行的排列在这三个轨道上. 因此, $\mathrm{Ni}_{2}^{+}$的基态为 ${ }^{4} \Sigma_{\mathrm{g}}$. 本文中采用反应物环己烷在气相中的 稳定构型——椅式构型, 反应物的前线分子轨道列于图 2 , (a)中环己烷的HOMO为三个轴 $\mathrm{H}$ 的 $1 \mathrm{~s}$ 轨道形成的三中 心离域键, (b) 中 ${ }^{4} \mathrm{Ni}_{2}^{+}$的LUMO为 $\sigma$ *轨道. 根据对称性匹 配原则, $\mathrm{Ni}_{2}^{+}$将会沿着三个轴氢方向进攻环己烷.

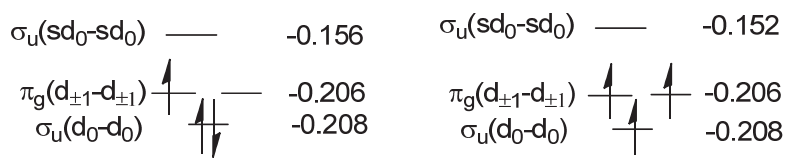

$$
\begin{aligned}
& \delta_{\mathrm{u}}\left(\mathrm{d}_{ \pm 2}-\mathrm{d}_{ \pm 2}\right) \text { 忛 } \frac{11}{4}-0.216 \quad \delta_{\mathrm{u}}\left(\mathrm{d}_{ \pm 2}-\mathrm{d}_{ \pm 2}\right) \frac{1}{1} \frac{11}{4}-0.219
\end{aligned}
$$

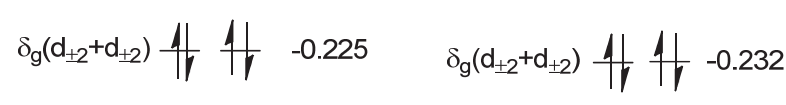

$$
\begin{aligned}
& \begin{array}{llllll}
\sigma_{\mathrm{g}}\left(\mathrm{d}_{0}+\mathrm{d}_{0}\right) \quad \text { H } & -0.238 & \sigma_{\mathrm{g}}\left(\mathrm{d}_{0}+\mathrm{d}_{0}\right) & \text { मी } & -0.248
\end{array}
\end{aligned}
$$

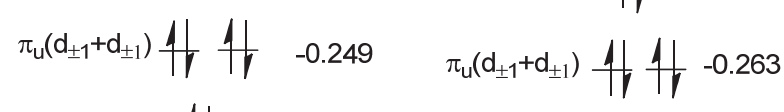

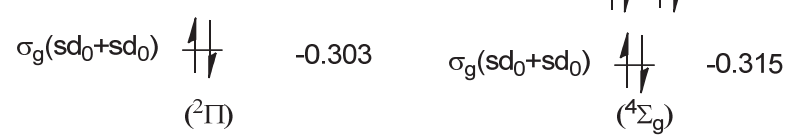

图 $1 \mathrm{Ni}_{2}^{+}$在二、四重态的分子轨道能级图(能量单位: Hartree)

Figure 1 Orbital diagram of quartet and doublet $\mathrm{Ni}_{2}^{+}$at the B3LYP level, MO energies (in Hartree) are calculated at the full valence (19electron/12orbital)
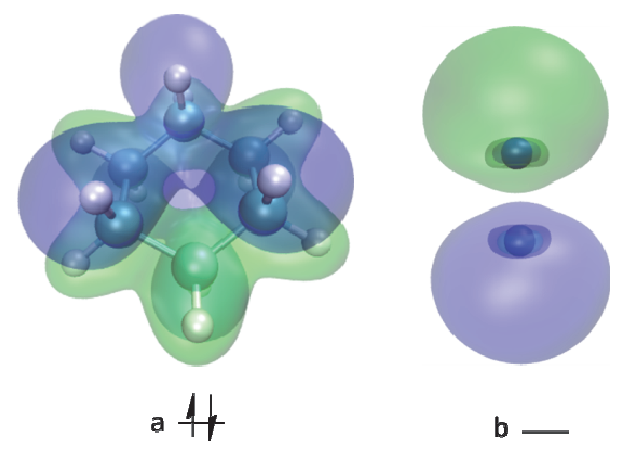

图 2 (a)环已烷的最高占据轨道 (HOMO); (b) ${ }^{4} \mathrm{Ni}_{2}^{+}$的最低空轨 (LUMO)

Figure 2 (a) HOMO of cyclohexane with fully occupied; (b) LUMO of ${ }^{4} \mathrm{Ni}_{2}^{+}$dimer $\sigma_{\mathrm{u}}$

\section{2 反应机理分析}

\subsection{1 环已烷的同面脱氢}

环己烷在二、四重态下同面脱氢的反应势能面见图 3 , 二、四重态下第一分子脱氢反应中各驻点的几何构型 列于图 4. 我们把 $\mathrm{Ni}_{2}^{+}+\mathrm{C}_{6} \mathrm{H}_{12} \rightarrow\left[\mathrm{Ni}_{2}\left(\mathrm{C}_{6} \mathrm{H}_{6}\right)\right]^{+}+3 \mathrm{H}_{2}$ 反应 分为两部分进行讨论, 第一部分为第一分子脱氢, 即反
应物至 ${ }^{2}$ IM5. 第二部分为第二、三分子脱氢. 即, ${ }^{2}$ IM5 至 ${ }^{2} \mathrm{P}$.

如图 3 所示, $\mathrm{Ni}_{2}^{+}$以四重态 $\left({ }^{4} \Sigma_{\mathrm{g}}\right)$ 进入反应通道, 与 $c-\mathrm{C}_{6} \mathrm{H}_{12}$ 相互作用时形成初始复合物 ${ }^{4} \mathrm{IM} 1$, 该复合物具 有 $46 \mathrm{kcal} / \mathrm{mol}$ 的稳定化能, 其中轴向的 C1-H11 (1.13 $\AA), \mathrm{C} 3-\mathrm{H} 31$ (1.13 $\AA), \mathrm{C} 5-\mathrm{H} 51$ (1.12 $\AA$ ) 比 $c-\mathrm{C}_{6} \mathrm{H}_{12}$ 中的 轴向 $\mathrm{C}-\mathrm{H}(1.10 \AA)$ 要长, $\mathrm{Ni}-\mathrm{Ni}$ 键的距离从 $2.16 \AA$ 变为 $2.34 \AA$, 说明在 $\mathrm{Ni}_{2}^{+}$与 $c-\mathrm{C}_{6} \mathrm{H}_{12}$ 之间存在 $\eta 3$ 配位. 二重态 下初始络合物 ${ }^{2} \mathrm{IM} 1$ 的稳定化能为 $35 \mathrm{kcal} / \mathrm{mol}$, 其中三 个轴向 $\mathrm{C}-\mathrm{H}$ 键分别为 $1.13,1.14$ 和 $1.13 \AA, \mathrm{Ni}-\mathrm{Ni}$ 键被 拉长 $1.11 \AA$. 表明四重态下 $\mathrm{Ni}_{2}^{+}$与 $c-\mathrm{C}_{6} \mathrm{H}_{12}$ 的 $\eta 3$ 配位要 强于二重态. 根据势能图 3, 反应在到达 ${ }^{4} \mathrm{TS} 1 / 2$ 前发生 了势能面交叉，运用在第二部分中提到的计算方法找到 了相应的 CP1 和 MECP1, 见支持信息图 S1(a). 经过 MECP1 后反应从四重态转化到二重态势能面形成过渡 态 ${ }^{2} \mathrm{TS} 1 / 2,{ }^{2} \mathrm{TS} 1 / 2$ 中 $\mathrm{H} 31$ 在 $\mathrm{C} 3$ 和 $\mathrm{Ni} 2$ 之间振动, 之后 $\mathrm{H} 31$ 迁移到 Ni1 和 Ni2 之间并形成氢桥键中间体 ${ }^{2} \mathrm{IM} 2$, ${ }^{2} \mathrm{IM} 2$ 通过 ${ }^{2} \mathrm{TS} 2 / 3$ 完成 H21 的迁移并形成双氢桥键的稳 定构型 ${ }^{2} \mathrm{IM} 3$ ，该中间体是整个反应势能面上的全局能 量最低点, 其相对能量为 $-52 \mathrm{kcal} / \mathrm{mol}$, 下一步经过 $\mathrm{H} 21$ 和 $\mathrm{H} 31$ 相互靠近的振动过渡态 ${ }^{2} \mathrm{TS} 3 / 4$ 形成氢分子 络合物 ${ }^{2} \mathrm{IM} 4, \mathrm{H}_{2}\left(\mathrm{Ni}_{2} \mathrm{C}_{6} \mathrm{H}_{10}\right)^{+}$, 该过程为第一分子脱氢的 决速步骤, 势垒为 $21 \mathrm{kcal} / \mathrm{mol}$, 最后脱去 $\mathrm{H} 21-\mathrm{H} 31$, 形 成 ${ }^{2} \mathrm{IM} 5,\left(\mathrm{Ni}_{2} \mathrm{C}_{6} \mathrm{H}_{10}\right)^{+}$. 与二重态不同, 四重态下双氢桥 键中间体 ${ }^{4} \mathrm{IM} 3$ 转为氢分子络合物 ${ }^{4} \mathrm{IM} 5$ 需要两步反应.

值得注意的是：(1) 在 ${ }^{4}$ IM5 $\rightarrow{ }^{4}$ IM6 过程中，体系的 能量下降了 $2 \mathrm{kcal} / \mathrm{mol}$, 我们考虑了两方面的因素来解 释这一现象, 第一, $\mathrm{Ni}-\mathrm{Ni}$ 键长从 $2.45 \AA$ 变为 $2.32 \AA$, 该过程放热 $4.26 \mathrm{kcal} / \mathrm{mol}$. 第二, 计算得出的 $\mathrm{H}_{2}$ 的解离 能为 $3.37 \mathrm{kcal} / \mathrm{mol}$, 综合这两方面的能量变化, 体系能 量总体下降 $0.89 \mathrm{kcal} / \mathrm{mol}$, 定性解释了这一现象. (2) 第 一分子脱氢过程中, 反应在经过 MECP1 后主要在二重 态势能面上发生，但是四重态势能面各驻点能量很大程 度的低于反应物, 最高势垒 ${ }^{4} \mathrm{TS} 2 / 3$ 也低于反应物 21 $\mathrm{kcal} / \mathrm{mol}$. 所以我们认为第一分子脱氢产物是两种自旋 态 ${ }^{2}$ IM5 和 ${ }^{4}$ IM6 的混合物, 其中以二重态产物 ${ }^{2}$ IM5 为 主.

二重态下 $\mathrm{Ni}_{2}^{+}$与 $c-\mathrm{C}_{6} \mathrm{H}_{2}$ 发生第二、三分子脱氢得到 的优化构型见图 5, 四重态下的优化构型见图 S2. 如图 5 所示, 在 ${ }^{2} \mathrm{IM} 5 \rightarrow^{2} \mathrm{TS} 5 / 13 \rightarrow{ }^{2} \mathrm{IM} 13$ 过程中, H11 从 C1 迁 移到 $\mathrm{Ni}_{2}^{+}$的桥位. 随后 ${ }^{2} \mathrm{IM} 13$ 通过 $\mathrm{TS} 13 / 14$ 发生了碳环 的异构化过程形成 ${ }^{2} \mathrm{IM} 14$, 该过程中 C4 从相对碳环较 高的位置调整到较低位使 H41 接近 Ni2, ${ }^{2} \mathrm{IM} 14$ 中 $\mathrm{C} 4-$ H41 (1.15 ̊) 已经被 Ni2 略微活化. 与二重态不同的是, 四重态下 $\mathrm{H} 11$ 的迁移需要两步才能完成(见图 S2), 即 ${ }^{4} \mathrm{IM} 6 \rightarrow{ }^{4} \mathrm{TS} 6 / 14 \rightarrow{ }^{4} \mathrm{IM} 14 \rightarrow{ }^{4} \mathrm{TS} 14 / 15 \rightarrow{ }^{4} \mathrm{IM} 15$. 在反应到 达 ${ }^{4} \mathrm{IM} 16$ 之前, 发生了两个势能面的交叉, 得到的 


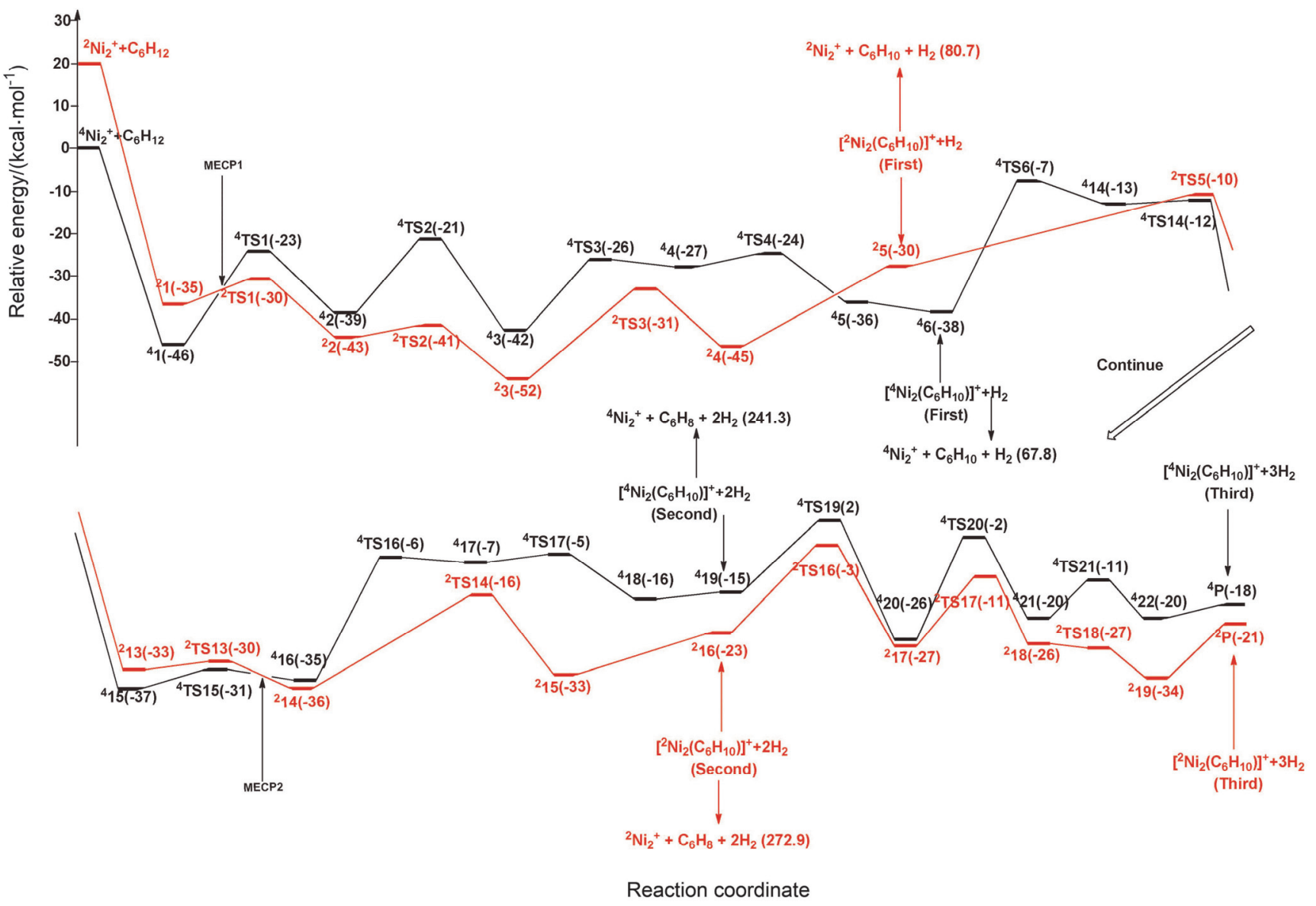

图 3 反应 $\mathrm{Ni}_{2}^{+}+\mathrm{C}_{6} \mathrm{H}_{12} \rightarrow \mathrm{Ni}_{2} \mathrm{C}_{6} \mathrm{H}_{6}^{+}+3 \mathrm{H}_{2}$ 在二、四重态下同面脱氢势能图, 红色实线表示二重态, 黑色实线表示四重态, 为简便起见, 将中间体用 ${ }^{2} 1$ 表示, 过渡态表示为 ${ }^{2} \mathrm{TS} 1$, 以此类推(相对能包含了零点能校正, 能量单位: $\mathrm{kcal} / \mathrm{mol}$ )

Figure 3 Potential energy surfaces of the reaction $\mathrm{Ni}_{2}^{+}+\mathrm{C}_{6} \mathrm{H}_{12} \rightarrow \mathrm{Ni}_{2} \mathrm{C}_{6} \mathrm{H}_{6}^{+}+3 \mathrm{H}_{2}$ in quartet (black continuous lines) and doublet (red continuous lines) states and numbers in parentheses are the relative energies $(\mathrm{kcal} / \mathrm{mol})$ after zero-point energy correction at the B3LYP level

$\mathrm{CP} 2$ 和 MECP2 列于图 S1(b). 该 MECP2 使四重态中间 体完全转化为二重态, 此后的反应将一直在二重态势能 面上进行. 之后, ${ }^{2} \mathrm{IM} 14$ 经过 ${ }^{2} \mathrm{TS} 14 / 15$ 发生 $\mathrm{H} 41$ 的迁移 形成氢分子络合物 ${ }^{2} \mathrm{IM} 15, \mathrm{H}_{2}\left(\mathrm{Ni}_{2} \mathrm{C}_{6} \mathrm{H}_{8}\right)^{+}$, 并最终脱去 $\mathrm{H} 11-\mathrm{H} 41$, 形成 ${ }^{2} \mathrm{IM} 16,\left(\mathrm{Ni}_{2} \mathrm{C}_{6} \mathrm{H}_{8}\right)^{+}$. 即, 第二分子脱氢 产物是二重态. 紧接着, 环己烷继续发生第三分子脱氢, 从 ${ }^{2} \mathrm{IM} 16$ 到 ${ }^{2} \mathrm{IM} 17$ 完成 $\mathrm{H} 61$ 从 C6 到 $\mathrm{Ni}_{2}^{+}$的转移, ${ }^{2} \mathrm{IM} 17$ 到 ${ }^{2} \mathrm{IM} 19$ 完成 $\mathrm{H} 51$ 从 C5 到 $\mathrm{Ni}_{2}^{+}$的转移, 最终形成产物 ${ }^{2} \mathrm{IM} 20$. 对 ${ }^{2} \mathrm{IM} 20$ 进行 NBO 分析, 发现 Ni1, Ni2 的空 $\mathrm{d}_{x 2-\mathrm{y} 2}$ 轨道分别接受了来自 $\mathrm{C} 1-\mathrm{C} 6, \mathrm{C} 3-\mathrm{C} 4$ 上的 $\pi$ 键电 子, 并把各自的 $\mathrm{d}_{x y}$ 电子反馈给它们的 $\pi^{*}$ 轨道形成反馈 $\pi$ 键, 这种反馈 $\pi$ 键增强了 $\mathrm{Ni}_{2}^{+}$与 $\mathrm{C}_{6} \mathrm{H}_{6}$ 之间的结合, 使 $\mathrm{Ni}_{2}^{+}$与 $\mathrm{C}_{6} \mathrm{H}_{6}$ 的解离能高达 $84.9 \mathrm{kcal} / \mathrm{mol}$, 该产物在室温 下不能发生进一步解离.

在第二、三分子脱氢中, 四重态势能面各驻点能量 略低于反应物, 而且四重态下反应势垒普遍较高, 这对 于反应是非常不利的, 反应在经过 MECP2 后完全转化 为二重态, 所以第二、三分子脱氢产物均为二重态.

结合反应势能面图 3, 构型图 4, 图 5 将第一分子脱
氢和第二、三分子脱氢进行对比, 发现以下异同点: (1) 第一分子脱氢的势能面普遍低于第二、三分子脱氢，第 一分子脱氢放热 $20 \mathrm{kcal} / \mathrm{mol}$, 产物为二、四重态的混合 物，主要以二重态为主. 第二、三分子脱氢分别放热 23 $\mathrm{kcal} / \mathrm{mol}, 21 \mathrm{kcal} / \mathrm{mol}$, 产物为二重态. (2) 发生第一分子 脱氢时, $\mathrm{Ni}_{2}^{+}$与环己烷处于近似 “垂直” 的相对位置, 而 二、三分子脱氢时, $\mathrm{Ni}_{2}^{+}$与环平面保持 “平行” 。这种 现象可能是由于 $c-\mathrm{C}_{6} \mathrm{H}_{10}$ 中 $\pi$ 键电子与 $\mathrm{Ni}_{2}^{+}$的空 $\mathrm{d}_{x y}$ 轨道 之间的相互作用所致. (3) 对第一分子脱氢而言, 决速 步骤是氢分子络合物的形成 $\left({ }^{2} \mathrm{IM} 3 \rightarrow^{2} \mathrm{TS} 3 / 4 \rightarrow{ }^{2} \mathrm{IM} 4\right)$, 势 垒为 $19 \mathrm{kcal} / \mathrm{mol}$. 第二、三分子脱氢时, 决速步骤为第 一个 $\mathrm{H}$ 原子的迁移 $\left({ }^{2} \mathrm{IM} 5 \rightarrow{ }^{2} \mathrm{TS} 5 / 13 \rightarrow{ }^{2} \mathrm{IM} 13,{ }^{2} \mathrm{IM} 16 \rightarrow\right.$ $\left.{ }^{2} \mathrm{TS} 16 / 17 \rightarrow{ }^{2} \mathrm{IM} 17\right)$, 势垒均为 $20 \mathrm{kcal} / \mathrm{mol}$.

\subsection{2 环已烷的异面脱氢}

为了解释异面脱氢现象, 实验 ${ }^{[8]}$ 提出了一种翻转机 理, 即, 在第一分子脱氢之后, $\mathrm{Ni}_{2}^{+}$活化了环己烯中的 一个 $\mathrm{C}-\mathrm{C} \sigma$ 键, 并通过 $\sigma$ 键的断裂使 $\mathrm{Ni}_{2}^{+}$翻转至环已烯 反应面 2, 再发生第二、三分子脱氢, 我们对该机理进行 了理论计算, 图 6 列出了翻转过程的势能面. 图 7 为二 

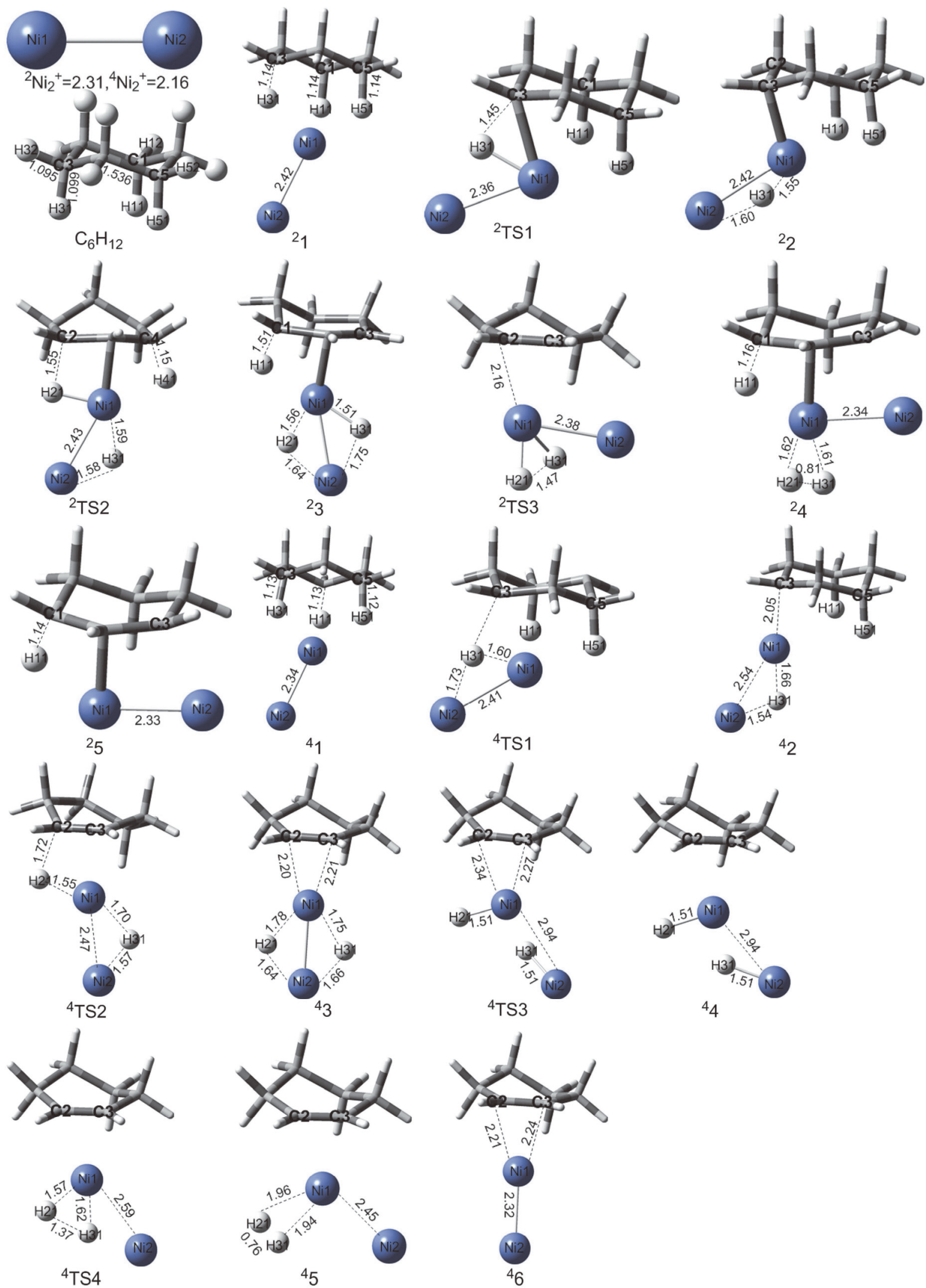

图 4 二、四重态下第一分子脱氢反应中各驻点的几何构型, 中间体表示为 ${ }^{2} 1$, 过渡态表示为 ${ }^{2} \mathrm{TS} 1$, 以此类推(键长单位: $\AA$ )

Figure 4 Optimized geometries for the stationary points of the reaction $\mathrm{Ni}_{2}^{+}+\mathrm{C}_{6} \mathrm{H}_{12} \rightarrow \mathrm{Ni}_{2} \mathrm{C}_{6} \mathrm{H}_{10}^{+}+\mathrm{H}_{2}$ in two spin states (bond lengths in angstroms) 


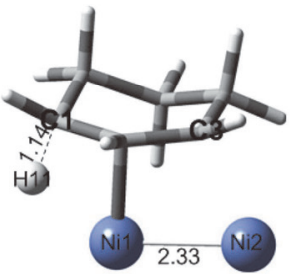

25
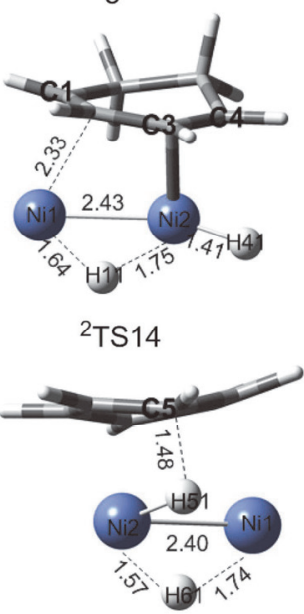

${ }^{2}$ TS17

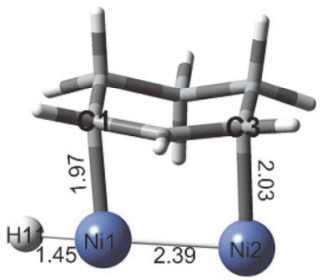

${ }^{2} \mathrm{TS} 5 / 13$

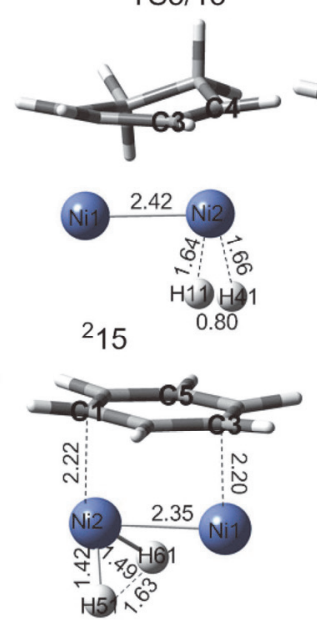

218

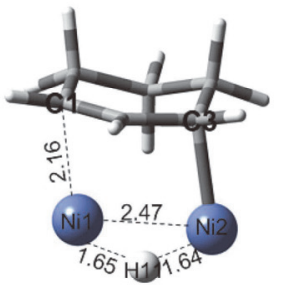

213

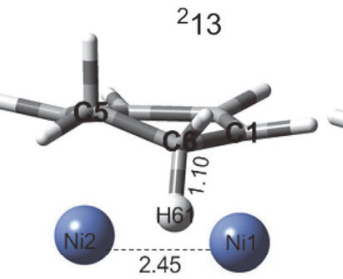

216
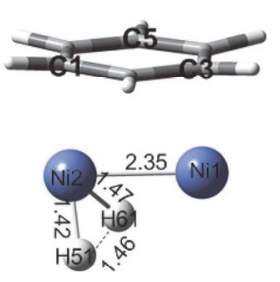

${ }^{2}$ TS18

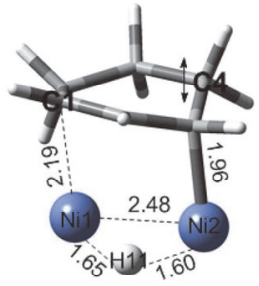

${ }^{2} \mathrm{TS} 13$

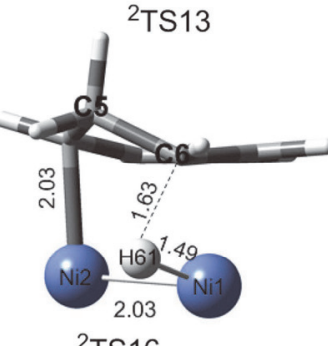

${ }^{2}$ TS16

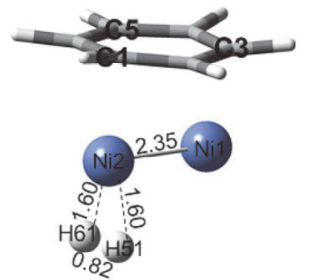

219

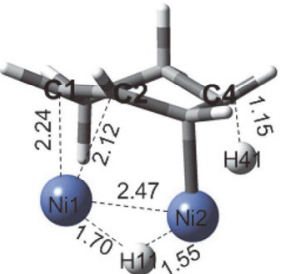

214
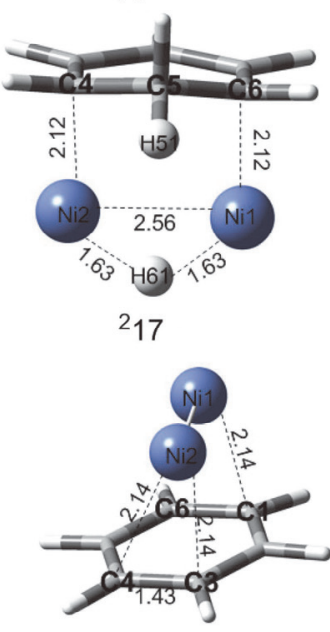

${ }^{2} 20$

图 5 二重态下发生第二、三分子脱氢的各驻点的几何构型, 中间体表示为 ${ }^{2} 5$, 过渡态表示为 ${ }^{2} \mathrm{TS} 13$, 以此类推(键长单位: $\AA$ )

Figure 5 Optimized geometries for the stationary points of the reaction $\left[\mathrm{Ni}_{2}\left(\mathrm{C}_{6} \mathrm{H}_{10}\right)\right]^{+} \rightarrow \mathrm{Ni}_{2} \mathrm{C}_{6} \mathrm{H}_{6}^{+}+2 \mathrm{H}_{2}$ in doublet state (bond lengths in angstrom)

重态下的优化构型, 四重态优化构型见图 S3.

翻转过程总结为以下四步:

(A) $\mathrm{Ni}_{2}^{+}$插入 $\mathrm{C}-\mathrm{C} \sigma$ 键 $\left({ }^{4} \mathrm{IM} 5 \rightarrow{ }^{2} \mathrm{TS} 5 / 6 \rightarrow{ }^{2} \mathrm{IM} 6\right)$. 环 己烷的翻转过程开始于第一分子脱氢产物 ${ }^{4} \mathrm{IM} 6$, 在反应 到达 ${ }^{4} \mathrm{TS} 6 / 7$ 之前, 发生了四重态和二重态的交叉, 降低 了反应的势垒, 反应由四重态转为二重态并形成过渡态 ${ }^{2} \mathrm{TS} 5 / 6,{ }^{2} \mathrm{TS} 5 / 6$ 中 $\mathrm{C} 4$ 和 $\mathrm{C} 5$ 振动模式为伸缩振动, 表明 $\mathrm{Ni} 2$ 已经插入 4 , C5之间, 最后形成 ${ }^{2} \mathrm{IM} 6$. CP3及MECP3见 图S1(c).

(B) H51 从 C5 到 C2 的迁移 $\left({ }^{2} \mathrm{IM} 6 \rightarrow{ }^{2} \mathrm{TS} 6 / 7 \rightarrow{ }^{2} \mathrm{IM} 7\right.$ $\left.\rightarrow^{2} \mathrm{TS} 7 / 8 \rightarrow{ }^{2} \mathrm{IM} 8\right)$. 第一步反应 ${ }^{2} \mathrm{IM} 6 \rightarrow^{2} \mathrm{TS} 6 / 7 \rightarrow{ }^{2} \mathrm{IM} 7$ 与上 述 ${ }^{2} \mathrm{IM} 13 \rightarrow{ }^{2} \mathrm{TS} 13 / 14 \rightarrow{ }^{2} \mathrm{IM} 14$ 类似, 也是碳环自身的调 整, ${ }^{2} \mathrm{IM} 7$ 中 5 - $\mathrm{H} 51$ (1.13 $\AA$ ) 被拉长, Ni1与H51的距离为 $1.89 \AA$. 预示着H51将会在C5与Ni1之间发生迁移. 第二 步反应 ${ }^{2} \mathrm{IM} 7 \rightarrow^{2} \mathrm{TS} 7 / 8 \rightarrow{ }^{2} \mathrm{IM} 8$ 过程中, H51通过一步反应 从 $\mathrm{C} 5$ 转移到 $\mathrm{C} 2$, 使 $\mathrm{C} 2$ 从 $\mathrm{sp}^{2}$ 杂化转化为 $\mathrm{sp}^{3}$ 杂化, $\mathrm{C} 2, \mathrm{C} 3$ 之间变为 $\sigma$ 键, 由于该步骤包含了一步完成的氢迁移过 程, 所以势垒较高为 $29 \mathrm{kcal} / \mathrm{mol}$, 与二重态不同的是, 中间体 ${ }^{4}$ IM7 (见支持信息图S3)不需要调整自身构型就 可以直接发生H51的迁移，但是H51迁移却是两步反应， 即, ${ }^{4} \mathrm{IM} 7 \rightarrow{ }^{4} \mathrm{TS} 7 / 8 \rightarrow{ }^{4} \mathrm{IM} 8$ 完成了 $\mathrm{H} 51$ 从 5 到 $\mathrm{Ni} 1$ 的迁移, ${ }^{4} \mathrm{IM} 8 \rightarrow{ }^{4} \mathrm{TS} 8 / 9 \rightarrow{ }^{4} \mathrm{IM} 9$ 完成了 $\mathrm{H} 51$ 从Ni1到 $\mathrm{C} 2$ 的迁移.

(C) $\mathrm{C} 2-\mathrm{C} 3 \sigma$ 键的旋转 $\left({ }^{2} \mathrm{IM} 8 \rightarrow^{2} \mathrm{TS} 8 / 9 \rightarrow{ }^{2} \mathrm{IM} 9 \rightarrow\right.$ $\left.{ }^{2} \mathrm{TS} 9 / 10 \rightarrow{ }^{2} \mathrm{IM} 10\right)$. 该过程中 ${ }^{2} \mathrm{IM} 9 \rightarrow{ }^{2} \mathrm{TS} 9 / 10 \rightarrow{ }^{2} \mathrm{IM} 10$ 为翻 转过程的决速步骤, 过渡态 ${ }^{2} \mathrm{TS} 9 / 10$ 的势垒为 33 $\mathrm{kcal} / \mathrm{mol}$, 吸热 $3 \mathrm{kcal} / \mathrm{mol}$. NBO分析表明, 这个较大的 势垒是由于 在 ${ }^{2} \mathrm{IM} 9$ 中 $\mathrm{Ni1}$ 的 $\mathrm{d}_{ \pm 1}$ 轨道与 $\mathrm{C} 3-\mathrm{C} 4$ 的 $\pi$ 键之间 存在很强的配位作用.

(D) 翻转产物 ${ }^{4} \mathrm{IM} 13$ 的形成 $\left({ }^{2} \mathrm{IM} 10 \rightarrow{ }^{2} \mathrm{TS} 10 / 11 \rightarrow\right.$ $\left.{ }^{4} \mathrm{IM} 12 \rightarrow{ }^{4} \mathrm{TS} 12 / 13 \rightarrow{ }^{4} \mathrm{IM} 13\right)$. 反 应 ${ }^{2} \mathrm{IM} 10 \rightarrow{ }^{2} \mathrm{TS} 10 / 11 \rightarrow$ ${ }^{4} \mathrm{IM} 12$ 为一个合环步骤, 该过程中 Ni1 与 $\mathrm{C} 3$ 结合, $\mathrm{C} 5$ 与 $\mathrm{C} 4$ 结合. 在 ${ }^{2} \mathrm{TS} 10 / 11 \rightarrow{ }^{2} \mathrm{IM} 11$ 过程中, 再次出现了势能面的 交叉使反应能垒大大降低, 之后反应由二重态转为四重 态并形成合环中间体 ${ }^{4} \mathrm{IM} 12, \mathrm{CP} 4$ 及 MECP4 见图 S1(d). 在 ${ }^{4} \mathrm{IM} 12 \rightarrow{ }^{4} \mathrm{TS} 12 / 13 \rightarrow{ }^{4} \mathrm{IM} 13$ (见图 S3) 过程中, 发生了 $\mathrm{H} 42$ 从 4 到 $\mathrm{C} 3$ 的迁移, 最终形成翻转产物 ${ }^{4} \mathrm{IM} 13$.

根据翻转过程势能面图6, 二、四重态构型图7, 图 S3. 我们将翻转过程简化图式2. 如图式2所示, 翻转开 始的中间体为四重态, 但翻转过程主要在二重态势能面 进行, 最终又转为四重态翻转产物 ${ }^{4} \mathrm{IM} 13 .{ }^{4} \mathrm{IM} 13$ 与翻转 开始中间体 ${ }^{4} \mathrm{IM} 6$ 有同样的构型和能量, 翻转过程中 $\mathrm{Ni}_{2}^{+}$ 从碳环的面 1 翻转到面 2 , 翻转产物 ${ }^{4} \mathrm{IM} 13$ 随后会发生第 二、三分子脱氢反应. 如图3所示, ${ }^{4}$ IM13 会沿着 ${ }^{4}$ IM6 的 反应路径: ${ }^{4} \mathrm{IM} 13\left({ }^{4} \mathrm{IM} 6\right) \rightarrow{ }^{4} \mathrm{TS} 6 / 14 \rightarrow{ }^{4} \mathrm{IM} 14 \rightarrow{ }^{4} \mathrm{TS} 14 / 15$ $\rightarrow{ }^{4} \mathrm{IM} 15 \rightarrow{ }^{4} \mathrm{TS} 15 / 16 \rightarrow \mathrm{CP} 2 \rightarrow{ }^{2} \mathrm{IM} 14$. 反应在经过MECP2 之后转化为二重态, 并最终生成产物 ${ }^{2} \mathrm{P}$. 


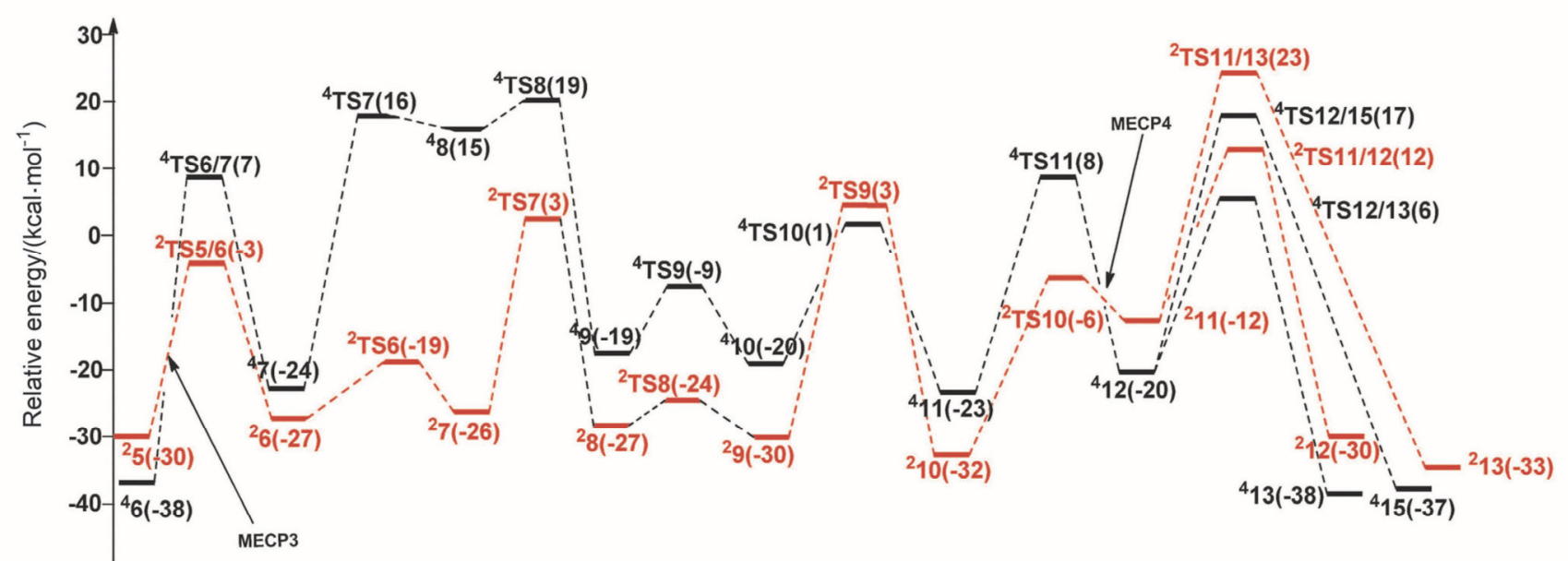

Reaction coordinate

图 6 二、四重态下翻转过程势能图, 红色虚线表示二重态, 黑色虚线表示四重态, 中间体表示为 ${ }^{2} 5$, 过渡态表示为 ${ }^{2} \mathrm{TS} 5$, 以此类推(相对能包含 了零点能校正, 能量单位: $\mathrm{kcal} / \mathrm{mol}$ )

Figure 6 Potential energy surfaces of flip process for doublet (red dashed lines) and quartet (black dashed lines) and numbers in parentheses are the relative energies $(\mathrm{kcal} / \mathrm{mol})$ after zero-point energy correction at the B3LYP level

\subsection{3 理论计算和实验的对比}

计算结果表明, 环己烷的第一分子脱氢是强放热过 程, 放热 $30 \mathrm{kcal} / \mathrm{mol}$. 决速步骤过渡态的相对能量为 $-30 \mathrm{kcal} / \mathrm{mol}$, 势垒为 $19 \mathrm{kcal} / \mathrm{mol}$. 该反应可以看作为 无势垒反应, 可以很快转向第二、第三分子脱氢. 所以 在实验中没有检测到第一分子脱氢产物 $\left[\mathrm{H}_{2}\right]$ 和 $\left[\mathrm{D}_{2}\right]$.

如果 $\mathrm{Ni}_{2}^{+}$从 ${ }^{2} \mathrm{IM} 5 /{ }^{4} \mathrm{IM} 6$ 或者 ${ }^{2} \mathrm{IM} 16 /{ }^{4} \mathrm{IM} 19$ 解离, 然后 进攻碳环的反应面 2, 也可以得到这些异面脱氢产物 $\left[\mathrm{H}_{2}, \mathrm{D}_{2}\right],\left[\mathrm{H}_{4}, \mathrm{D}_{2}\right]$ 和 $\left[\mathrm{H}_{2}, \mathrm{D}_{4}\right]$, 这两种情况所需的解离能列 于势能图 3, 二、四重态下 $\mathrm{Ni}_{2}^{+}$从环已烯解离所需的解离 能分别为 80.7 和 $67.8 \mathrm{kcal} / \mathrm{mol}, \mathrm{Ni}_{2}^{+}$从环已二烯解离所 需的解离能分别为 272.9 和 $241.3 \mathrm{kcal} / \mathrm{mol}$. 由于较高的 解离能, 排除了这种解离脱氢的可能性, 异面脱氢现象 只能通过本文中计算的翻转机理来解释.

本文中还考虑了 $[\mathrm{H}-\mathrm{D}]$ 混合脱去的可能性, 在所有 中间体中, ${ }^{4} \mathrm{IM} 11\left({ }^{2} \mathrm{IM} 12\right)$ 是在整个翻转过程中唯一可能 导致 [H-D]混和的中间体. 该过程的优化构型列于支持 材料图 S4, 势能图列于图 6. [H-D] 混合是指 ${ }^{2} \mathrm{IM} 11 /{ }^{4} \mathrm{IM} 12$ 中, 环己烷反应面 1 的 $\mathrm{H} 41$ 原子直接从 $\mathrm{C} 4$ 转移到 $\mathrm{Ni}_{2}^{+}$形成 ${ }^{2} \mathrm{IM} 13 /{ }^{4} \mathrm{IM} 15$ (见图 S4), 紧接着, ${ }^{2} \mathrm{IM} 13 /{ }^{4} \mathrm{IM} 15$ 脱去反应面 2 的 $\mathrm{H} 22$, 从而形成氢气分子 [H41，H22](两个 $\mathrm{H}$ 原子来自环己烷两个面). 然而该 [H-D]混合过程在二、四重态下的过渡态相对能量分别 为 23 和 $17 \mathrm{kcal} / \mathrm{mol}$, 均高于二、四重态下 ${ }^{2} \mathrm{IM} 11 /{ }^{4} \mathrm{IM} 12$ 中的 $\mathrm{H} 42$ 原子从 $\mathrm{C} 4$ 重排到 $\mathrm{C} 3$ 形成 ${ }^{2} \mathrm{IM} 12 /{ }^{4} \mathrm{IM} 13$ 时过 渡态的能量 ${ }^{2} \mathrm{TS} 11 / 12$ (12 kcal/mol), ${ }^{4} \mathrm{TS} 12 / 13$ (6 $\mathrm{kcal} / \mathrm{mol}$ ), 所以反应会沿着最低反应通道 ${ }^{4} \mathrm{IM} 12 \rightarrow{ }^{4} \mathrm{TS} 12 / 13 \rightarrow{ }^{4} \mathrm{IM} 13$ 发生, 不会脱去 $[\mathrm{H}-\mathrm{D}]$, 即, 氢 气分子的形成均来自环已烷的同一面. 在实验 ${ }^{[8]}$ 中也未
检测到 $\left[\mathrm{H}_{3}, \mathrm{D}\right],\left[\mathrm{D}_{3}, \mathrm{H}\right],\left[\mathrm{H}_{5}, \mathrm{D}\right],\left[\mathrm{H}, \mathrm{D}_{5}\right]$ 和 $\left[\mathrm{H}_{3}, \mathrm{D}_{3}\right]$ 脱氢产物. 对比二、四同重态下同面脱氢和异面脱氢的优化构 型, 可以得到一些值得参考的规律性: (1) 对二、四重态 而言, 当碳环脱第一个 $\mathrm{H}$ 原子时 $\left({ }^{2} \mathrm{IM} 2,{ }^{2} \mathrm{IM} 3,{ }^{2} \mathrm{IM} 13\right.$, ${ }^{2} \mathrm{IM} 17,{ }^{4} \mathrm{IM} 2,{ }^{4} \mathrm{IM} 3,{ }^{4} \mathrm{IM} 15$ 和 $\left.{ }^{4} \mathrm{IM} 20\right), \mathrm{H}$ 总是迁移至 $\mathrm{Ni}_{2}^{+}$ 的桥位, 形成氢桥键. 这是由于 $\mathrm{Ni}_{2}^{+}$的分子轨道中存在 $\mathrm{d}_{ \pm 1}$ 轨道，在 $\mathrm{Ni} 1, \mathrm{Ni} 2$ 形成的 $\mathrm{d}_{ \pm 1}$ 轨道中，电子云集中于 两金属之间并与键轴成 $45^{\circ}$ 夹角的位置. 当发生第一个 氢迁移时, $\mathrm{Ni}_{2}^{+}$的 $\mathrm{d}_{ \pm 1}$ 轨道很容易接受 $\mathrm{H}$ 的 $1 \mathrm{~s}$ 电子, 形 成稳定的角形 d-s-d 氢桥键. (2) 二重态下氢迁移是一步 反应, 如 ${ }^{2} \mathrm{IM} 3 \rightarrow \mathrm{IM} 4,{ }^{2} \mathrm{IM} 7 \rightarrow{ }^{2} \mathrm{IM} 8,{ }^{2} \mathrm{IM} 5 \rightarrow{ }^{2} \mathrm{IM} 13$ 和 ${ }^{2} \mathrm{IM} 14 \rightarrow{ }^{2} \mathrm{IM} 15$. 而在四重态下氢迁移需要两步才能完 成 $\left({ }^{4} \mathrm{IM} 3 \rightarrow{ }^{4} \mathrm{IM} 4 \rightarrow{ }^{4} \mathrm{IM} 5, \quad{ }^{4} \mathrm{IM} 7 \rightarrow{ }^{4} \mathrm{IM} 8 \rightarrow{ }^{4} \mathrm{IM} 9, \quad{ }^{4} \mathrm{IM} 6 \rightarrow\right.$ ${ }^{4} \mathrm{IM} 14 \rightarrow{ }^{4} \mathrm{IM} 15$ 和 $\left.{ }^{4} \mathrm{IM} 16 \rightarrow{ }^{4} \mathrm{IM} 17 \rightarrow{ }^{4} \mathrm{IM} 18\right)$. 通过分析这 些中间体的 NBO 以及自旋密度(表 1)发现，中间体 ${ }^{4} \mathrm{IM} 3,{ }^{4} \mathrm{IM} 7,{ }^{4} \mathrm{IM} 6$ 和 ${ }^{4} \mathrm{IM} 16$ 中的自旋单电子几乎平均分 布在 $\mathrm{Ni} 1, \mathrm{Ni} 2$ 上，在 $\mathrm{H}$ 迁移过程中，当 $\mathrm{H}$ 靠近 $\mathrm{Ni}_{2}^{+}$中的 $\mathrm{Ni}$, Ni1 上的单电子更容易与 $\mathrm{H}$ 的 $1 \mathrm{~s}$ 电子自旋成对形 成 $\mathrm{Ni}-\mathrm{H} \sigma$ 键, 而且为了保持自旋守恒, 部分电子必须 跃迁到 $\sigma^{*}$ 反键轨道上, 因此在四重态中这些形成 $\mathrm{Ni}-\mathrm{H}$ $\sigma$ 键的中间体能量普遍都很高. 二重态下, 单电子主要 集中在 $\mathrm{Ni} 2$ 上, 发生氢迁移时, 氢原子更倾向于通过 $\mathrm{Ni} 1$ 接近 $\mathrm{Ni} 2$, 最终形成 $\mathrm{Ni} 1-\mathrm{H}-\mathrm{Ni} 2$ 氢桥键.

\section{3 最低能量交叉点(MECP)与自旋-轨道耦合(SOC)}

为了进一步剖析 MECP 处电子的排布和翻转, 运用 NBO 理论分析了 MECP1, MECP2, MECP3 和 MECP4 的前线分子轨道. 如图 8(a) 所示, $\varphi_{\mathrm{a} 1}$ 主要是由 $\mathrm{Ni}_{2}^{+}$的 $4 \mathrm{~s}$ 轨道组成, 它是二重态的 LUMO, 也是四重态的 HOMO. $\varphi_{c 1}$ 由 $\mathrm{Ni} 2$ 的 $3 \mathrm{~d}_{x y}$ 轨道组成, 它是二重态的最高 


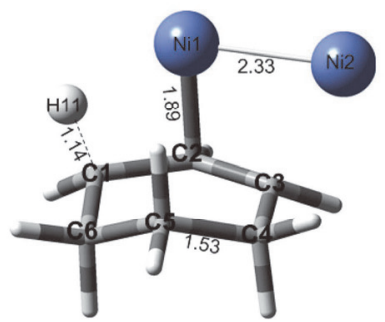

25

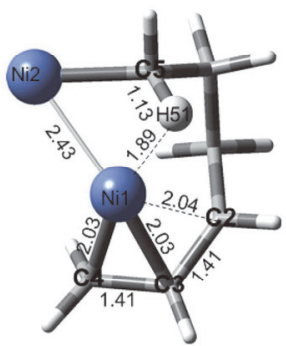

27

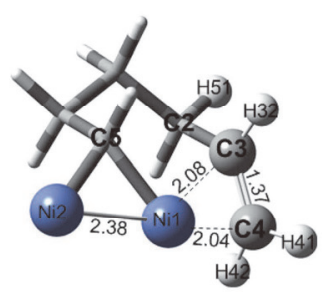

${ }^{2} 9$

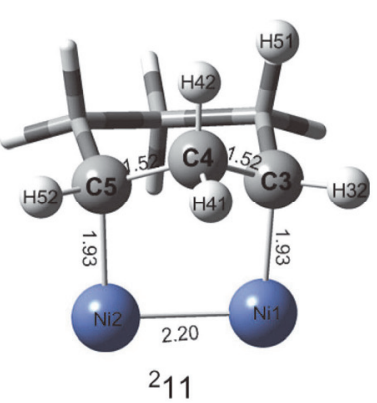

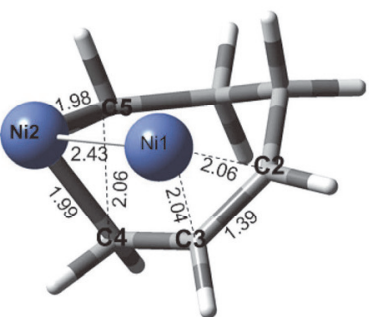

${ }^{2} \mathrm{TS} 5 / 6$

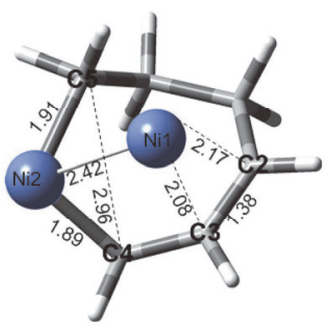

${ }^{2} 6$

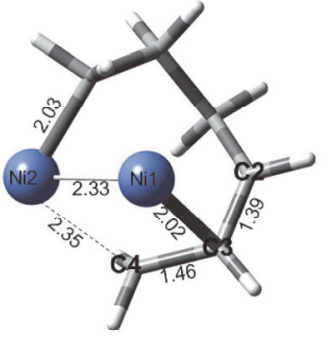

${ }^{2}$ TS6

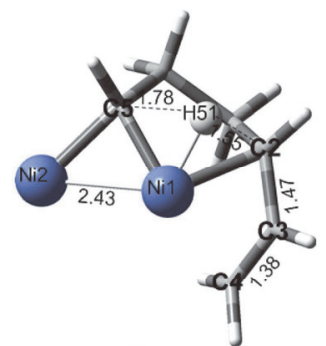

${ }^{2} \mathrm{TS} 7$

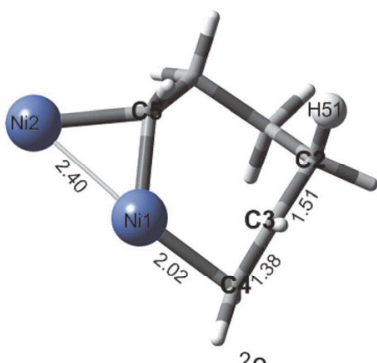

28

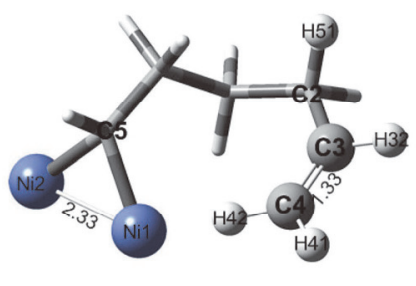

${ }^{2}$ TS9

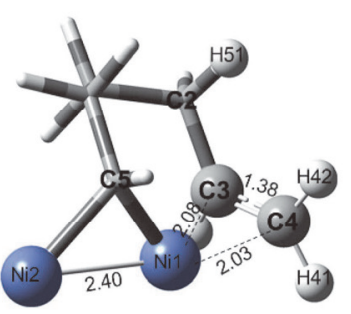

210

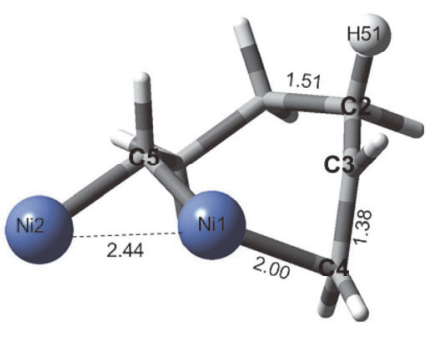

${ }^{2}$ TS8
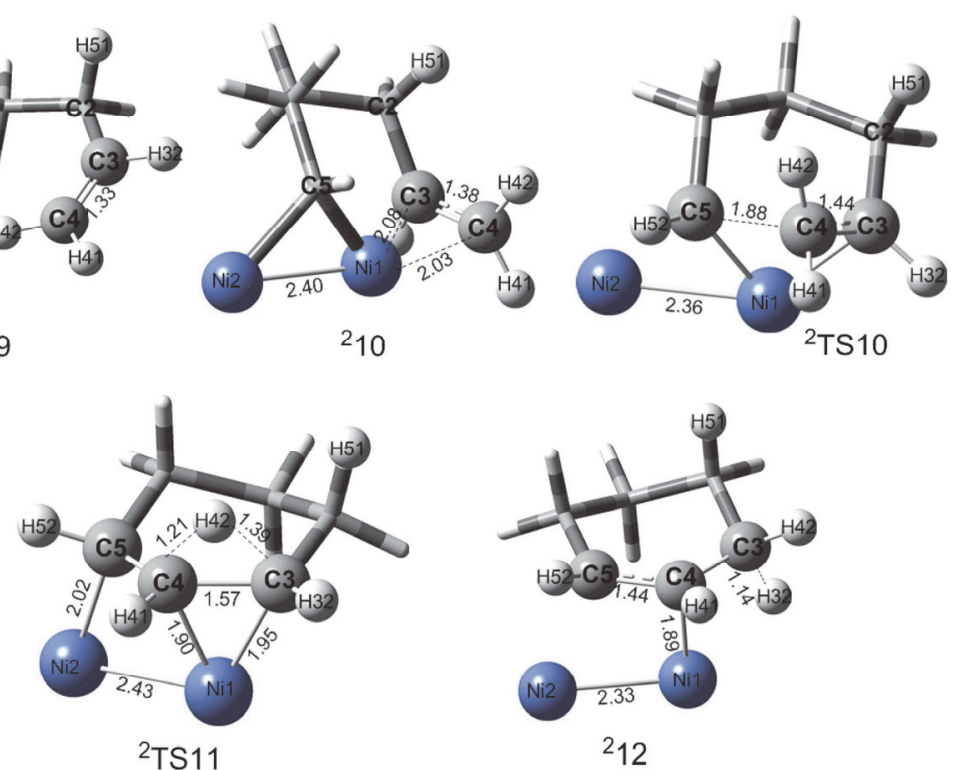

图 7 二重态下翻转过程各驻点的几何构型, 中间体表示为 ${ }^{2} 5$, 过渡态表示为 ${ }^{2} \mathrm{TS} 6$, 以此类推(键长单位: $\AA$ )

Figure 7 Optimized geometries for the stationary points of the flip process in doublet state (bond lengths in angstroms)

双占轨道, 也是四重态的最低单占轨道. 当反应进行到 MECP1 时, 会发生不同自旋态的混合. 此时, $\varphi_{\mathrm{a} 1}$ 中 Ni2 的 $4 \mathrm{~s}$ 轨道上单占据的 $\alpha$ 电子会翻转到 $\varphi_{\mathrm{c} 1}$ 的 $3 \mathrm{~d}_{x y}$ 轨道, 完成由四重态向二重态的跃迁. MECP2 的前线分子轨 道如图 8(b) 所示, $\varphi_{\mathrm{a} 2}$ 主要由 $\mathrm{Ni}_{2}^{+}$的 $\sigma^{*}$ 轨道和 $\mathrm{C} 1, \mathrm{C} 2$ 和 $\mathrm{C} 3$ 之间形成的共轭 $\pi$ *轨道正向重叠而成, $\varphi_{\mathrm{c} 2}$ 主要由 Ni1 的 $3 \mathrm{~d}_{x 2-y 2}$ 轨道组成, 在 MECP2 附近时, 填充在 $\varphi_{\mathrm{a} 2}$ 上的 $\alpha$ 电子翻转到 $\varphi_{c 2}$ 的 $3 d_{x 2-y 2}$ 轨道, 完成四重态向二重 态的跃迁. MECP3 的前线分子轨道 8(c) 与 $8(\mathrm{a})$ 类似, $\varphi_{\mathrm{a}} 3$ 主要由 $\mathrm{Ni} 2$ 的 $4 \mathrm{~s}$ 轨道组成, $\varphi_{\mathrm{c} 3}$ 中, Ni1 的 $3 \mathrm{~d}_{x 2-y 2}$ 轨道和 $\mathrm{C} 2, \mathrm{C} 3$ 的 $2 p$ 轨道头碰头形成两个 $\sigma$ 键, 在 MECP3 附近, $\varphi_{\mathrm{a} 3}$ 上的 $\alpha$ 电子会转移到 $\mathrm{Ni} 1$ 的 $3 \mathrm{~d}_{x 2-y 2}$ 轨道上, 并发生自 旋翻转与原有的 $\alpha$ 电子自旋配对, 最终完成四重态向二
重态的跃迁. MECP4 的前线分子轨道 8(d)与 $8(\mathrm{~b})$ 类似, $\varphi_{\mathrm{a} 4}$ 也是由 $\mathrm{Ni}_{2}^{+}$的 $\sigma^{*}$ 轨道和 $\mathrm{C} 5, \mathrm{C} 3$ 的 $2 p$ 轨道正向重叠而 成, $\varphi_{\mathrm{c} 4}$ 主要由 $\mathrm{Ni} 1, \mathrm{Ni} 2$ 的 $\mathrm{d}_{x z}$ 轨道组成, 与前三者不同的 是, 这次是 $\mathrm{Ni} 1, \mathrm{Ni} 2$ 上 $\mathrm{d}_{x z}$ 轨道中的 $\beta$ 电子翻转到 $\varphi_{\mathrm{a} 4}$, 完 成二重态到四重态的跃迁.

根据非绝热理论 ${ }^{[20]}$, 系间窝越过程中的自旋翻转 是轨道磁矩与自旋磁矩共同作用来完成的. 因此, 在发 生系间窝越的体系中，考虑非绝热态下的自旋-轨道耦 合作用是不可或缺的. 对于本文中的两态反应而言, 在 最低能量交叉点(MECP1 MECP4)能否发生有效的系 间窝越是反应的关键因素. 在 MECP 处不同自旋态之间 的能量和构型相同，反应体系实际是二、四重态的混合 态, 它们之间的耦合作用会使体系能量降低. 电子的自 


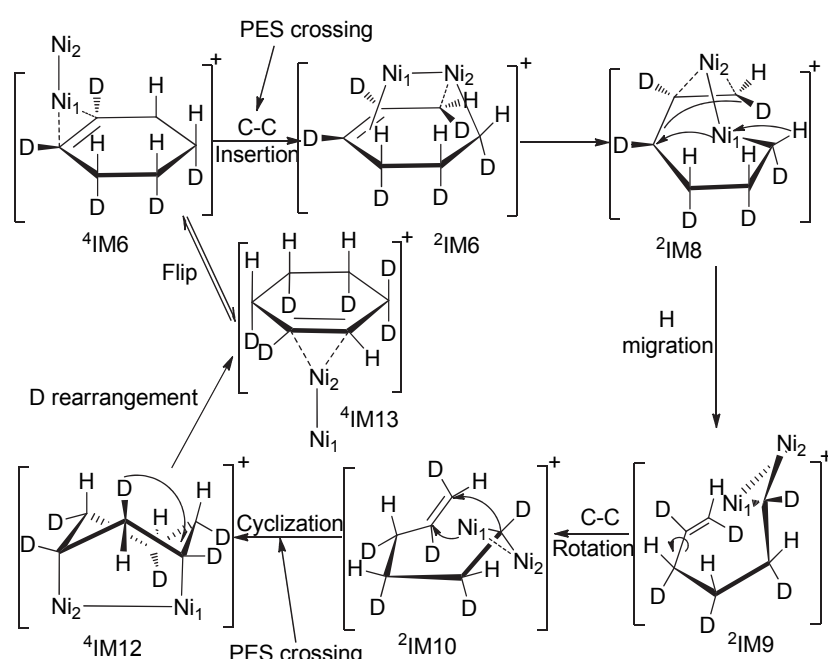

图式 2 翻转过程机理图

Scheme 2 The mechanism of flip process

PES crossing means the crossing of potential energy surfaces

表 1 部分中间体中 $\mathrm{Ni} 1, \mathrm{Ni} 2$ 的自旋密度

Table 1 The spin densities on Ni1, Ni2 in part of intermediates

\begin{tabular}{ccc}
\hline \multirow{2}{*}{ 中间体 } & \multicolumn{2}{c}{ 自旋密度 } \\
\cline { 2 - 3 } & $\mathrm{Ni}$ & $\mathrm{Ni} 2$ \\
\hline${ }^{2} \mathrm{IM} 3$ & -0.10 & 1.06 \\
${ }^{4} \mathrm{IM} 3$ & 1.19 & 1.35 \\
${ }^{2} \mathrm{IM} 5$ & -0.05 & 1.07 \\
${ }^{4} \mathrm{IM} 6$ & 1.14 & 1.83 \\
${ }^{2} \mathrm{IM} 7$ & 0.05 & 1.05 \\
${ }^{4} \mathrm{IM} 7$ & 1.23 & 1.39 \\
${ }^{2} \mathrm{IM} 14$ & -0.18 & 1.00 \\
${ }^{4} \mathrm{IM} 16$ & 1.25 & 1.25 \\
\hline
\end{tabular}

旋磁矩与轨道磁矩相互作用使不同自旋态势能面交叉 区发生电子的自旋翻转，自旋一轨道耦合常数越大，电 子的自旋翻转越容易发生. 根据单电子近似以及自旋一 轨道耦合 Hamiltonian 算符, 运用 GAMESS 程序, 计算 出了 $\mathrm{MECP} 1 \sim \mathrm{MECP} 4$ 处的自旋-轨道耦合常数(耦合非 对角元 $\mathrm{H}_{12}$ ), 其值分别为 $318.01 ， 396.89 ， 268.74$ 和 $306.67 \mathrm{~cm}^{-1}$. 较大的自旋-轨道耦合常数表明体系存在 强的自旋-轨道耦合作用, 即, 电子在最低能量交叉点 (MECP)处能发生有效地翻转, 使得反应体系从一个势 能面跃迁到另一个势能面，从而降低了反应的势垒 ${ }^{[22]}$.

\section{4 结论}

本文采用密度泛函 B3LYP 方法, 研究了两态反应 $\mathrm{Ni}_{2}^{+}$与 $c-\mathrm{C}_{6} \mathrm{H}_{12}$ 的反应机理以及自旋轨道耦合现象, 报 道了势能面上各反应物、中间体和过渡态的构型特征及 能量. 得出如下结论: $\mathrm{Ni}_{2}^{+}$与 $c-\mathrm{C}_{6} \mathrm{H}_{12}$ 的反应是一个非常 复杂的两态反应, 所有反应通道中包含了两次势能面的 交叉，并找着了它们各自对应的 MECP1 MECP4. 其 中, MECP1 MECP3 附近发生了四重态到二重态的交 叉，MECP4 附近是二重态到四重态的交叉，计算的 MECP1 MECP4 处的 SOC 值分别为 318.01，396.89, 268.74 和 $306.67 \mathrm{~cm}^{-1}$, 均大于 $100 \mathrm{~cm}^{-1}$, 进一步验证了 势能面在最低能量交叉点(MECP)处发生了有效地跃 迁. 对于反应通道的研究表明, 同面脱氢是主要反应通 道, 异面脱氢由于翻转过程中的决速步骤: ${ }^{2} \mathrm{IM} 9 \rightarrow{ }^{2} \mathrm{TS} 9 / 10 \rightarrow{ }^{2} \mathrm{IM} 10$ 需要克服 $33 \mathrm{kcal} / \mathrm{mol}$ 的势垒而 成为次要通道, 本研究能很好的解释 Schwarz 等的实验 现象. 总反应通道见图式 3.

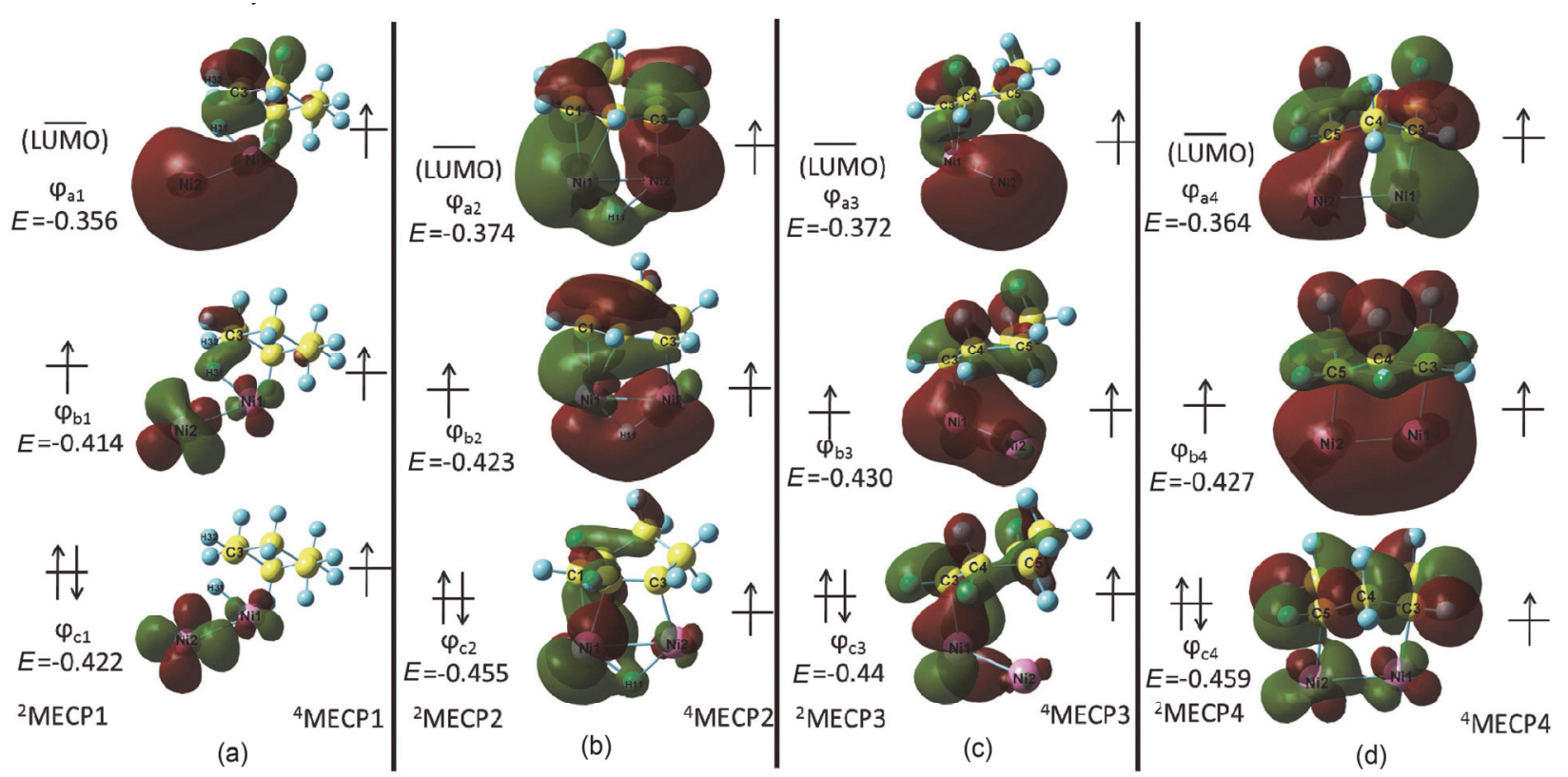

图 8 MECP1 (a), MECP2 (b), MECP3 (c)和 MECP4 (d)处的前线分子轨道图(能量单位: Hartree) Figure 8 FMO (frontier molecular orbital) of MECP1 (a), MECP2 (b), MECP3 (c) and MECP4 (d) 


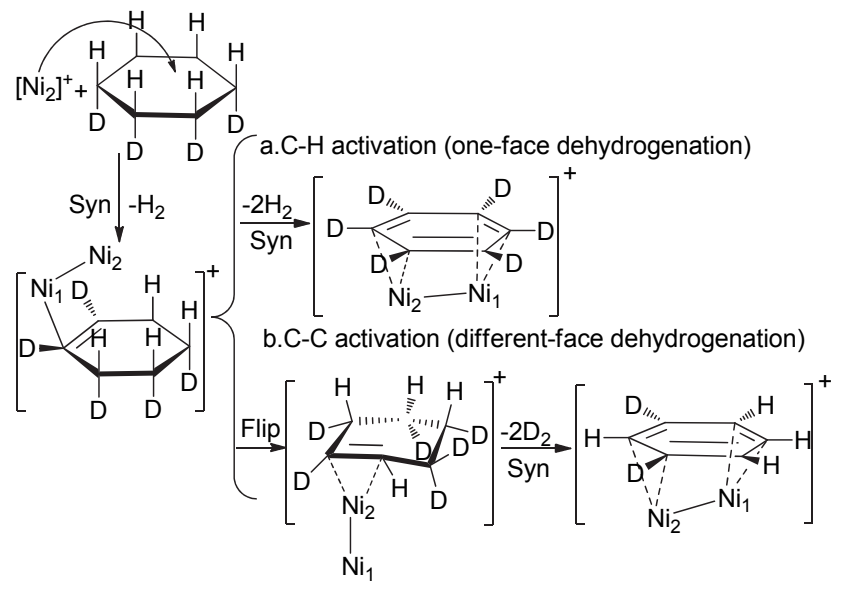

图式 3 总反应通道示意图

Scheme 3 The global reaction channels

\section{致谢}

感谢中国科学院寒区旱区环境与工程研究所超级 计算兰州分中心提供的计算资源.

\section{References}

[1] Tsuda, M.; Diño, W. A.; Nakanishi, H.; Watanabe, S.; Kasai, H. Jpn. J. Appl. Phys. 2005, 44, 402.
[2] Koel, B. E.; Blank, D. A.; Carter, E. A. J. Mol. Catal. A: Chem. 1998, 131, 39.

[3] Tsai, M. C.; Friend, C.; Muetterties, E. J. Am. Chem. Soc. 1982, 104, 2539

[4] Qi, S.; Huang, J.; Chen, H.; Gao, Z.; Yi, C.; Yang, B. Acta Chim. Sinica 2012, 70, 2467. (齐随涛, 黄俊, 陈昊, 高子丰, 伊春海, 杨 伯伦, 化学学报, 2012, 70, 2467.)

[5] Seemeyer, K. Organometallics 1995, 1995, 4465.

[6] Tsuda, M.; Agerico Diño, W.; Nakanishi, H.; Kasai, H. J. Phys. Soc. Jpn. 2004, 73, 1281.

[7] Geng, Z.; Wang, Y.; Wang, Y.; Liang, J.; Sheng, Y.; Sun, X. Acta Chim. Sinica 2010，68，391. (耿志远，王玉芝，王永成，梁俊胥， 盛玉, 孙小建, 化学学报, 2010, 68, 391.)

[8] Schlangen, M.; Schwarz, H. J. Catal. 2011, 284, 126.

[9] Becke, A. D. Phys. Rev. A 1988, 38, 3098.

[10] Becke, A. D. J. Chem. Phys. 1993, 98, 5648.

[11] Lee, C.; Yang, W.; Parr, R. G. Phys. Rev. B 1988, 37, 785

[12] Wadt, W. R.; Hay, P. J. J. Chem. Phys. 1985, 82, 284.

[13] Hay, P. J.; Wadt, W. R. J. Chem. Phys. 1985, 82, 299

[14] Hay, P. J.; Wadt, W. R. J. Chem. Phys. 1985, 82, 270

[15] Yoshizawa, K.; Shiota, Y.; Yamabe, T. J. Chem. Phys. 1999, 111, 538.

[16] Harvey, J. N.; Aschi, M.; Schwarz, H.; Koch, W. Theor. Chem. Acc. 1998, $99,95$.

[17] Frisch, M.; Trucks, G.; Schlegel, H.; Scuseria, G.; Robb, M.; Cheeseman, J.; Scalmani, G.; Barone, V.; Mennucci, B.; Petersson, G. Gaussian 09, Revision A. 02, Gaussian. Inc., Wallingford, 2009.

[18] Schmid, M.; Baldridge, K.; Boatz, J.; Elbert, S.; Gordon, M.; Jensen, J.; Koseki, S.; Matsunaga, N.; Nguyen, K.; Su, S. J. Comput. Chem. $1993,14,1347$.

[19] Danovich, D.; Shaik, S. J. Am. Chem. Soc. 1997, 119, 1773.

[20] Poli, R.; Harvey, J. N. Chem. Soc. Rev. 2003, 32, 1.

[21] Kemper, P. R.; Bowers, M. T. J. Phys. Chem. 1991, 95, 5134.

[22] Harvey, J. N. Phys. Chem. Chem. Phys. 2007, 9, 331. 